\title{
Preventing and Managing Cardiovascular Toxicities of Tyrosine Kinase Inhibitors in Chronic Myeloid Leukemia
}

\author{
Nahla AM Hamed* \\ Faculty of Medicine, Alexandria University, Egypt
}

Submission: March 20, 2018; Published: April 04, 2018

"Correspondence Address: Nahla AM Hamed, Professor of Hematology, Faculty of Medicine, Alexandria University, Egypt, Email: drhamedn@hotmail.com

\begin{abstract}
TKIs are small molecules that interfere with the kinase activity. The main TKIs currently used in the treatment of CML are imatinib, nilotinib, dasatinib, bosutinib and ponatinib. Each TKI has a unique side-effect profile, which determines the choice of TKI in a given patient. Recently, TKI-associated vascular adverse events, seen with some of the TKIs, have emerged as one of the most disturbing and poorly understood TKI side effects.
\end{abstract}

Abbreviation: TKIs: Tyrosine Kinase Inhibitors; ATP: Adenosine Triphosphate; PAOD: Peripheral Arterial Occlusive Disease; PDGF: PlateletDerived Growth Factor; CV: Cardiovascular; ABI: Ankle Brachial Index

\section{Introduction}

TKIs have very high affinity to the ATP binding pocket of the TKs. They act by inhibiting the transfer of a phosphate group from ATP to a tyrosine residue [1]. TKIs inhibit TKs in cancerous and non-cancerous cells. Their action on normal tissues explains their side effects; the most common side effects include diarrhea and rash. Although cardiac toxicity is less common, it is more serious and difficult to diagnose at early stages [1].

Cardiovascular side effects of TKIs are varied and have included heart failure, left ventricular dysfunction, conduction abnormalities, QT prolongation, acute coronary syndromes, myocardial injury, arterial thromboses, and hypertension. Overall, systolic dysfunction with resultant heart failure is one of the most common important side effects. This often occurs because pathways that induce the pathological survival and abnormal proliferation of cancer cells may also regulate the survival of normal cells, including cardiomyocytes [2].

\section{Cardiovascular Toxicities of the individual TKI}

The need to use TKIs on a long-term basis emphasizes the importance of knowing which agents are associated with cardiac effects and understanding the mechanisms that underlie that toxicity [2].

\section{Imatinib}

Imatinib mesylate targets Bcr-Abl (the fusion protein encoded by the Philadelphia chromosome), c-Kit (the stem cell factor receptor) and PDGF $\alpha$ and $\beta$ receptors [1]. Imatinib has a safe vascular profile. The positive vascular effect of imatinib can also be suggested by the positive effect of the drug in patients with pulmonary hypertension [3]. It has been suggested that only patients with a history of cardiac disease should have standard cardiac monitoring [1].

There are conflicting results from different studies regarding the cardiotoxicity of imatinib, it seems that the observed pathologic changes do not necessarily translate into clinically significant cardiac toxicity. Prospective clinical trials that will follow patients on imatinib will be able to identify those patients who are more susceptible to develop a cardiac toxic effect and take the appropriate measures to protect them. As most of these patients will be treated for months or even years, long-term observation is needed since, the toxicity profile may be different in this case [1].

\section{Nilotinib}

Nilotinib is an inhibitor of Bcr-Abl, c-Kit and PDGFR $\alpha$ and $\beta$ receptors. It is 30 -fold more potent than imatinib in vitro [1]. Nilotinib prolongs the QT interval, and sudden deaths have been reported rarely (www.fda.gov/cder/Offices/OODP/ whatsnew/nilotinib.htm) [2]. In addition, nilotinib may cause hyperglycemia, hypertension, hyperlipidemia and vascular events [4]. CV events (defined as ischemic heart disease, ischemic cerebrovascular events and peripheral arterial disease) were reported in $7.5 \%$ of patients randomized to nilotinib $300 \mathrm{mg}$ twice daily and in $13.4 \%$ of patients randomized to nilotinib 
$400 \mathrm{mg}$ twice daily, compared with $2.1 \%$ of patients randomized to imatinib $400 \mathrm{mg}$ daily. Interestingly, CV events occurred more frequently in patients with a higher CV risk profile [4].

The participants of three phase III retrospective trials with different TKIs are divided into three groups: patients who received imatinib, patients who received nilotinib and those who received no TKI. The analysis revealed decreased rates of PAOD in the imatinib treated patients as compared to patients who were not treated with a TKI, and nilotinib treated patients had similar rates of PAOD as the no TKI group [3].

\section{Dasatinib}

Dasatinib is a TKI against Bcr-Abl, cKit, PDGFR- $\alpha$ and $\beta$ and the Src family of kinases. It is 300 -fold more potent than imatinib in vitro [1]. Dasatinib is associated with fluid retention including pericardial and pleural effusions. However, the mechanism has yet to be clarified. Dasatinib has also been implicated in the development of heart failure with up to $4 \%$ of patients in some series developing congestive heart failure (dasatinib prescribing information; https://www.sprycell. com/pdf/pi.pdf) [2]. Dasatinib may also cause pulmonary hypertension (5\%) and vascular events (include coronary, cerebral and peripheral events) [4].

\section{Bosutinib}

The contemporary data on vascular toxicity of bosutinib is scarce. A recent meta-analysis comparing TKIs and imatinib as a reference therapy showed no increased vascular toxicity with bosutinib. It should be kept in mind, that bosutinib inhibits some of the pathways inhibited by ponatinib, such as the SRC family kinases. A recently published meta-analysis has shown increased pooled incidence of cardiovascular events with bosutinib compared with imatinib and dasatinib. However, this meta-analysis was performed on non-randomized data, with only one of the included study evaluated bosutinib [3].

\section{Ponatinib}

Retrospective analysis of the PACE database suggests a higher risk of $\mathrm{CV}$ events in ponatinib, in a dose-dependent manner, in patients with CV risk factors or CV disease, as well as a signal for CV events. In addition, $\geq 26 \%$ of patients developed hypertension after initiating ponatinib, which may have contributed to CV events. These observations have prompted other studies to test lower doses of ponatinib in the hope of minimizing toxicity [4].

\section{The Molecular Mechanisms that Underlie Cardiovascular Risk}

In addition to imatinib, dasatinib and nilotinib also target Abl/Arg (Abl-related gene), PDGFRs, and c-Kit. Dasatinib, also targets the Src family. Targeting these pathways in cancer cells may inherently lead to on-target cardiotoxicity, manifested as cardiomyopathy, because of inhibition of these same prosurvival kinases in normal cardiomyocytes [2].
The role of ABL in cardiomyocytes is not clear [1]. Imatinib and nilotinib are type II inhibitors that bind 2 different regions on the kinase: the ATP pocket and an adjacent region that is accessible only when the kinase is inactive. Type II inhibitors thus bind and lock kinases in an inactive state. However, type II agents still typically inhibit $\geq 3$ kinases [2]. It has been found that JNK activation may be responsible for the cardiac toxicity observed with imatinib and inhibition of the JNK pathway markedly reduces the collapse of the mitochondrial membrane potential and cell death in adult cardiac myocytes [1]. The implication of dasatinib in the development of heart failure could certainly be due to inhibition of Abl. The inhibition of Src and a number of other kinases by dasatinib also could play a role as well [2].

\section{Preventing and Managing Cardiovascular Toxicities in the CML Population Treated with} TKIs

Clinical trials have revealed unanticipated side effects of targeted therapies involving the heart and other organs. Cardiovascular side effects have, in general, been able to be managed medically and typically have not prevented their use [2]. With a broad population of patients being treated with TKIs as well as increased patient survival and delayed tumor progression from improvements in cancer therapy, prevention and management of TKI-associated CV adverse events will become increasingly important [5].

\section{Some Clinical Recommendations for CML patients on TKIs Treatment}

At baseline (prior to treatment start), all patients should be assessed for CV disease by history and physical examination. In addition, basic CV risk factors such as blood pressure, fasting glucose, and fasting lipids should be monitored. In follow-up (1 month and 3 months), CV symptoms should be further assessed (and treated if necessary). CV risk factors should be further assessed at 3 months [4].

\section{Practical ABCDE steps to reduce CV disease in CML patients on TKI treatment [4]}

A: Awareness of CV disease signs and symptoms, Aspirin in selected cases, and ABI measurement should be considered in patients treated with nilotinib or ponatinib at baseline and follow-up to monitor for peripheral artery disease.

B: Blood pressure control.

C: Cigarette and tobacco cessation, Cholesterol level regular monitoring and treatment if needed.

D: Diabetes mellitus regular monitoring and treatment if needed, Diet and weight management

E: Exercise. 


\section{Cancer Therapy \& Oncology International Journal}

\section{Considerations for the Monitoring and Prevention of TKI-Associated Prolonged QT Intervals [5]:}

i. Avoid concomitant QT-prolonging drugs, including certain antiarrhythmic (e.g. amiodarone), antibiotic (e.g. clarithromycin), antifungal agents and non essential dietary and herbal supplements.

ii. Treatment should not be started in patients with QT interval $(>500 \mathrm{~ms})$.

iii. Electrocardiogram monitoring for QT intervals at baseline and frequently thereafter as indicated by the drug's prescribing information.

iv. Diarrhea may increase QT prolongation risk due to the resulting electrolyte imbalance; therefore, electrolytes should be monitored and diarrhea appropriately managed.

v. Potassium and magnesium levels should be monitored and supplemented as needed.

vi. TKI dosage should be modified or reduced in patients with impaired renal and hepatic function.

NB: A patient who has high CV risk (either CV disease at baseline or multiple cardiac risk factors) and who would benefit from a high-CV-risk TKI (e.g., ponatinib) is in need of more thorough assessment for $\mathrm{CV}$ issues and, if necessary, referred to a cardiologist [4].

\section{Conclusion}

Further prospective studies with objective cardiac monitoring and long-term follow-up are needed to define the incidence, natural history and risk factors for developing cardiotoxicity associated with the newer TKI more accurately.

\section{References}

1. Orphanos GS, Ioannidis GN, Ardavanis AG (2009) Cardiotoxicity induced by tyrosine kinase inhibitors. Acta Oncologica 48(7): 964-970

2. Chen MH, Kerkelä R, Force T (2008) Mechanisms of cardiac dysfunction associated with tyrosine kinase inhibitor cancer therapeutics. Circulation 118(1): 84-95.

3. Pasvolsky O, Leader A, Iakobishvili Z, Wasserstrum Y, Kornowski R et al. (2015) Tyrosine kinase inhibitor associated vascular toxicity in chronic myeloid leukemia. Cardio-Oncology 1:5.

4. Barber MC, Mauro MJ, Moslehi J (2017) Cardiovascular care of patients with chronic myeloid leukemia (CML) on tyrosine kinase inhibitor (TKI) therapy. ASH Education Book 2017(1): 110-114.

5. Lenihan DJ, Kowey PR (2013) Overview and management of cardiac adverse events associated with tyrosine kinase inhibitors. Oncologist 18(8): 900-908.

This work is licensed under Creative

DOI: 10.19080/CTOIJ.2018.10.555779
Your next submission with Juniper Publishers will reach you the below assets

- Quality Editorial service

- Swift Peer Review

- Reprints availability

- E-prints Service

- Manuscript Podcast for convenient understanding

- Global attainment for your research

- Manuscript accessibility in different formats ( Pdf, E-pub, Full Text, Audio)

- Unceasing customer service

Track the below URL for one-step submission https://juniperpublishers.com/online-submission.php 